\title{
Coronavirus disease 2019 (COVID-19): two case reports from a family cluster
}

\author{
Lei Tang ${ }_{1}^{1,2 \#}$, Zheng Ye ${ }^{1 \#}$, Zixing Huang ${ }^{1}$, Xianchun Zeng ${ }^{2}$, Tao Wang ${ }^{2}$, Rui Xu' ${ }^{2}$, Rongpin Wang ${ }^{2}$, \\ Bin Song
}

${ }^{1}$ Department of Radiology, West China Hospital, Sichuan University, Chengdu, China; ${ }^{2}$ Department of Radiology, Guizhou Provincial People's Hospital, Key Laboratory of Intelligent Medical Imaging Analysis and Accurate Diagnosis of Guizhou Province, International Exemplary Cooperation Base of Precision Imaging for Diagnosis and Treatment, Guiyang, China

\#These authors contributed equally to this work.

Correspondence to: Bin Song. Department of Radiology, West China Hospital, Sichuan University, No. 37 Guoxue Alley, Wuhou District, Chengdu 610041, China. Email: songlab_radiology@163.com.

\begin{abstract}
The coronavirus disease 2019 (COVID-19) is a new infectious disease, firstly appeared in Wuhan city and has rapidly spread to 114 countries outside China, which is receiving worldwide attention. As two important means of examination, computed tomography (CT) and real-time reverse transcription polymerase chain reaction (RT-PCR) have always been controversial in the clinical diagnosis of COVID-19 pneumonia. Here, we report a family cluster case of a father and a son diagnosed as COVID-19 at our hospital, and described the clinical manifestations, laboratory results, CT changes, diagnosis and treatment strategy of these two patients. Focus on the value of these two methods in the diagnosis and treatment of diseases, as well as their respective deficiencies. For patient 1 (father), the efficacy of RT-PCR is not satisfactory either in terms of diagnosis or follow-up, which may cause misdiagnosis and delay treatment. For patient 2 (son), the clinical symptoms were not obvious, but CT imaging clearly displayed dynamic changes of the lung lesions. Meanwhile, the two patients respectively underwent five chest CT examinations during their hospitalization and discharge follow-up, showing the potential harm of radiation. Therefore, in clinical work, doctors should make full use of the advantages of CT and RT-PCR, and take other measures to make up for their disadvantages.
\end{abstract}

Keywords: Coronavirus disease 2019 (COVID-19); coronavirus; pneumonia; case report

Submitted Feb 13, 2020. Accepted for publication Mar 13, 2020.

doi: 10.21037/apm.2020.03.34

View this article at: http://dx.doi.org/10.21037/apm.2020.03.34

\section{Introduction}

In December 2019, a series of pneumonia cases with unknown causes appeared in Wuhan, a densely populated city in central China, and shortly spread to other areas and countries within a month (1-4). With viral genome sequencing, scientists identified the pathogen as a novel coronavirus, and World Health Organization (WHO) officially named this disease as coronavirus disease 2019 (COVID-19) and the virus as severe acute respiratory syndrome coronavirus 2 (SARS-CoV-2) on February 11 2020 (5). Until March 12 2020, over 118,326 people were infected with 4,292 deaths in the world (6).

Clinically, real-time reverse transcription polymerase chain reaction (RT-PCR) of SARS-CoV-2 nucleic acid is the reference standard in diagnosing COVID-19, however, RT-PCR is a sophisticated technique with certain false negative rate $(7,8)$. Computed tomography $(\mathrm{CT})$ can be used for early diagnosis, disease assessment and follow-up, but cannot be the gold standard for diagnosis due to the lack of etiological evidence. There has always been a dispute between the two diagnostic methods. Here, we report a family cluster case, in which the father underwent RT-PCR multiple times but it was difficult to confirm the diagnosis, and the son presented mild clinical symptoms but significant CT manifestations. At the same time, the value of CT and RT-PCR in diagnosis and treatment was analyzed for better clinical guidance. We present the following cases in accordance with the CARE Guideline. 


\begin{tabular}{|c|c|c|c|c|c|c|c|c|c|c|c|c|c|c|c|c|c|c|c|c|}
\hline & \multirow{2}{*}{$\begin{array}{l}\text { Wuhan } \\
\text { BT }\end{array}$} & \multicolumn{19}{|c|}{ Guiyang } \\
\hline & & $37.5^{\circ} \mathrm{C}$ & & & & $38.8^{\circ}$ & & & & & & orma & & & & & & & & \\
\hline [1] & 22 & CT1 & 24 & 25 & 26 & 27 & 28 & СT2 & 30 & СТ3 & 1 & 2 & 3 & 4 & CT4 & 6 & 7 & 8 & 9 & CT5 \\
\hline
\end{tabular}

[2]

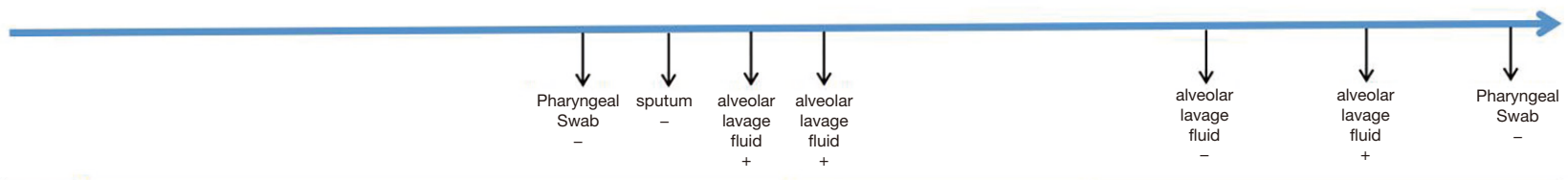

[3]

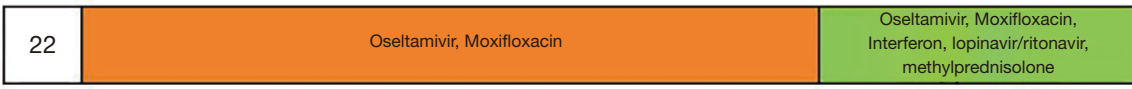

Oseltamivir, Moxifloxacin, Interferon, lopinavir/
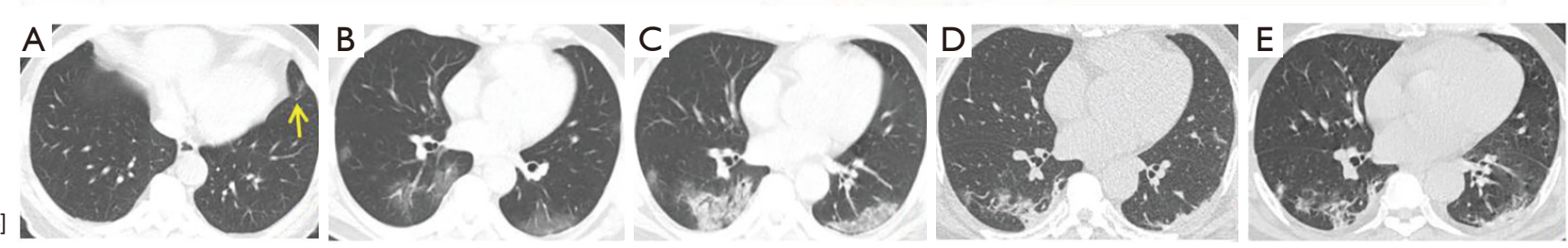

Figure 1 Time line and clinical data of patient 1 (father). [1] Dates filled in green are the dates on which two patients had close contacts while dates filled in yellow are the hospitalization dates. [2] Real-time reverse transcription polymerase chain reaction (RT-PCR). +, positive; -, negative. [3] Treatment. [4] CT images in the time course. BT, body temperature; CT, computed tomography.

\section{Case presentation}

A 53-year-old man (patient 1), a local driver in Wuhan, travelled to Guiyang city for a family reunion on January 22, 2020 and presented cough and fever $\left(37.5^{\circ} \mathrm{C}\right)$ the day after. He had a history of hypertension (max $150 / 98 \mathrm{mmHg}$ ) and took nifedipine orally (specific dose unknown). The patient received laboratory tests and first chest CT examination on January 23, which showed less lymphocyte count $\left(0.81 \times 10^{9} / \mathrm{L}\right.$, normal range: $\left.1.1 \times 10^{9}-3.2 \times 10^{9} / \mathrm{L}\right)$, elevated C-reactive protein $(11.04 \mathrm{mg} / \mathrm{L}$; normal range: $0-5 \mathrm{mg} / \mathrm{L})$ and erythrocyte sedimentation rate $(22 \mathrm{~mm} / \mathrm{h}$; normal range: $2-20.9 \mathrm{~mm} / \mathrm{h}$ ), as well as a few fibrosis foci in the inferior lingular segment of upper lobe of left lung on CT (Figure 1), then he was treated with oseltamivir (75 mg each time, 2 times daily) and moxifloxacin $(0.4 \mathrm{~g}$ each time, once daily). Because of the persistent fever (max $38.8^{\circ} \mathrm{C}$ ), the patient was admitted to the Guizhou Provincial People's Hospital on January 29 and had his second CT examination, which demonstrated multiple scattered ground glass opacities (GGO) in both lungs (Figure 1). However, the RT-PCR assay of the patient's nasopharyngeal swab on that day and sputum of the following day were negative for SARS-CoV-2 nucleic acid. On January 31 (the 12th day of onset), the third chest CT scan showed more advanced bilateral pulmonary foci with consolidation and air bronchi signs (Figure 1). Meanwhile, the RT-PCR for SARS-CoV-2 was positive in alveolar lavage fluid for 2 consecutive times, arterial partial oxygen pressure $\left(\mathrm{PO}_{2}\right)$ /oxygen absorption concentration $\left(\mathrm{FiO}_{2}\right) \leq 300 \mathrm{mmHg}$, and the patient was finally diagnosed with COVID-19 (severe type). After receiving lopinavir/ritonavir $(200 \mathrm{mg} / 50 \mathrm{mg}, 2$ tablets at a time, once a day) and interferon (50 $\mu \mathrm{g}$, spray inhalation, 2 times daily) antivirus treatment, with a small dose of methylprednisolone (40 mg, intravenous drip, once daily), his body temperature back to normal on February 2. On February 5 (the 17th day of onset), the fourth CT suggested partial absorption of pulmonary lesions, with a few fibrosis (Figure 1). Methylprednisolone was discontinued and other treatment regimens remained unchanged. In the following 5 days, RT-PCR was reviewed for 3 times (1 day interval), which revealed that the first alveolar lavage fluid was negative, the second alveolar lavage fluid was positive, and the third pharyngeal swab was negative. On February 10 , the fifth follow-up CT indicated continued absorption of the lesions (Figure 1), the patient's condition was stable and there was no special discomfort. In accordance with the requirements of the local epidemic monitoring and management department, he was transferred to a designated hospital for centralized treatment.

The son of patient 1, a 25-year-old Guiyang native (patient 2), had never been to Wuhan before he became ill, and had no contact with people from Wuhan except his parents. $\mathrm{He}$ developed fever $\left(37.8^{\circ} \mathrm{C}, 100.0^{\circ} \mathrm{F}\right)$ and cough on January 26 
Guiyang

\begin{tabular}{|c|c|c|c|c|c|c|c|c|c|c|c|c|c|c|c|c|c|c|c|c|c|c|c|c|c|c|c|c|c|c|c|c|}
\hline BT & & & & $37.8^{\circ}$ & ${ }^{\mathrm{C}} \mathrm{n}$ no & mal & & & & & & & & & & & & & & & & & & & & & & & & & & \\
\hline 22 & 23 & 24 & 25 & 26 & 27 & 28 & CT1 & 30 & 31 & Ст2 & 2 & 3 & 4 & СТ3 & 6 & 7 & 8 & CT4 & 10 & 11 & 12 & 13 & 14 & \begin{tabular}{l|l}
415 \\
4
\end{tabular} & 16 & 17 & 18 & 19 & 20 & 21 & \begin{tabular}{l|l}
1 & 22
\end{tabular} & \begin{tabular}{|l|l|}
23 & CT5 \\
\end{tabular} \\
\hline
\end{tabular}

[2]

[3]

\begin{tabular}{|c|c|c|c|c|c|c|c|c|c|c|c|}
\hline 22 & 23 & 24 & 25 & 26 & 27 & 28 & 29 & $\begin{array}{l}\text { Oseltamivir, Moxifloxacin, } \\
\text { Interferon, lopinavir/ritonavir }\end{array}$ & $\begin{array}{l}\text { Oseltamivir, Moxifloxacin, Interferon, } \\
\text { lopinavir/ritonavir, Methylprednisolone, } \\
\text { Thymosin, Hydrochloride abidor }\end{array}$ & $\begin{array}{l}\text { Oseltamivir, Moxifloxacin, Interferon, } \\
\text { lopinavir/ritonavir, Thymosin, } \\
\text { Hydrochloride abidor }\end{array}$ & 11 \\
\hline
\end{tabular}

[4]

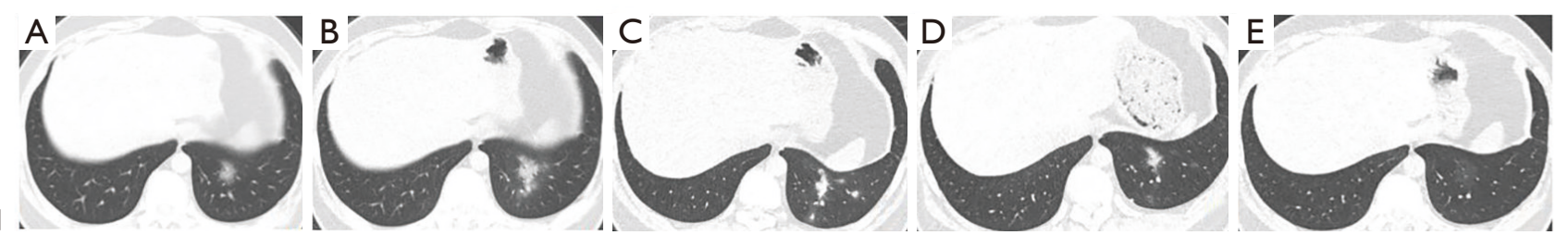

Figure 2 Time line and clinical data of patient 2 (son). [1] Dates filled in green are the dates on which two patients had close contacts, in yellow are the hospitalization dates, while in white are discharge dates. [2] Real-time reverse transcription polymerase chain reaction (RTPCR). +, positive; -, negative. [3] Treatment. [4] CT images in the time course. BT, body temperature; CT, computed tomography.

(after 4-day close contacts with his father). His mother was not infected. The patient was admitted on January 29, when laboratory tests only showed lymphocytopenia (lymphocyte count $1.10 \times 10^{9} / \mathrm{L}$ ) while chest CT revealed two regions of GGO in the middle lobe of the right lung and lower lobe of the left lung, mainly distributed in the subpleural area (Figure 2). Nasopharyngeal swabs for two consecutive times were positive for SARS-CoV-2 on RT-PCR and he was diagnosed with COVID-19. After taking lopinavir/ritonavir (200 mg/50 mg, 2 tablets at a time, once a day), the patient experienced mild dyspnea, nausea and increased bowel movements. On February 1, the patient was stable with normal body temperature and slightly larger left lung opacity in chest CT (Figure 2). On February 5, the third CT scan showed that the lesions in the lower lobe of left lung were smaller than before, and there was a new nodule shadow in the middle lobe of the right lung (Figure 2). At the same time, $\mathrm{CD} 3+, \mathrm{CD} 4+$, and CD4/CD8 were declined, suggesting decreased immunity and disease progression. Thymosin (80 mg, intravenous drip, once daily ) was added to enhance cellular immunity, hydrochloride abidor $(0.2 \mathrm{~g}$, three times per day) for antiviral treatment, and methylprednisolone sodium succinate (1st day-60 mg; 2 nd, 3rd day- $40 \mathrm{mg}$; 4th day- $20 \mathrm{mg}$; intravenous drip, once daily) for anti-inflammatory treatment. On February 9, the fourth chest CT suggested lung lesions were smaller than before (Figure 2), and the hormone was discontinued. He was discharged on February 10, because two pharyngeal swab RT-PCR tests (1 day interval) were negative. After discharge, the patient was quarantined at home for another 14 days. On February 24, his follow-up CT examination showed obvious absorption of bilateral lung lesions (Figure 2).

\section{Discussion}

The COVID-19 is a new infectious disease with fever, fatigue and dry cough as main clinical symptoms (9). Based on the current epidemiological investigation, the main transmission means are respiratory droplet and personto-person contact, and the incubation period is 1-14 days, mostly 3-7 days. Previous investigation confirmed asymptomatic people can also be the infection source and the existence of family cluster phenomenon $(10,11)$. In most COVID-19 patients, the laboratory tests show normal or decreased peripheral blood white blood cells, decreased while lymphocytes, elevated C-reactive protein and elevated erythrocyte sedimentation rate (11). Additionally, the chest CT images often show small patches and interstitial changes in the early stage, which were more obvious in the peripheral lung (12-14). In the progressive stage, multiple GGO and consolidation could occur in both lungs.

In early February, some medical personnel working in the frontline recommended CT as the standard while other doctors argue that all infectious diseases must be diagnosed 
with a pathogen, sparking a heated debate within Chinese medical community. In the Diagnosis and Treatment Plan of Novel Coronavirus Pneumonia (5th edition) released on February 5, highly suspected patients with positive CT manifestation have been regarded as the clinical confirmed cases in Hubei Province because of the false negative rate of RT-PCR analysis and shortage of the assay kits (11). As a result of the change in diagnostic criteria, the number of cases confirmed by CT increased significantly. However, due to the lack of specificity of CT findings, it was still difficult to guide treatment and follow-up without etiological evidence. Then, the use of CT as the confirmed diagnostic criterion was eliminated in the 6th edition of the protocol (15). Yet, the debate about the diagnostic efficacy of CT and RT-PCR still exists in the current epidemic prevention and control work.

The chronology of symptom onset and examination results is demonstrated in Figures 1,2. In this family cluster case with a father and a son, both of them should be considered as highly suspected cases according to the history of the epidemic, blood changes and chest CT images. In this case, patient 1 (the father) had more serious symptoms, increased C-reactive protein, and extensive radiological ground-glass lung lesions. However, his first two RT-PCR of nasopharyngeal swab and sputum were negative for SARS-CoV-2 nucleic acid. The diagnosis was not made until the third and fourth alveolar lavage fluid RT-PCR tests were positive, and the interval was 3 days. After treatment, RT-PCR results of three reexaminations (one day apart) showed that the first alveolar lavage fluid was negative, the second alveolar lavage fluid positive, and the third pharyngeal swab negative. As far as patient 1 is concerned, the efficacy of RT-PCR is not satisfactory either in terms of diagnosis or follow-up, which may cause misdiagnosis and delay treatment.

Previous studies have shown that false negatives and false positives of RT-PCR may be related to viral load and specimen contamination (8). The three pharyngeal swabs and sputum of patient 1 were all negative, and the positive rate of alveolar lavage fluid was higher, which may be related to the sampling site. Therefore, for patients with high suspicion of COVID-19 in clinical and CT imaging, multi-site sampling can be attempted, especially for the secretion of the lower respiratory tract, which can improve the diagnostic accuracy. Moreover, serological diagnostic criteria (positive for COVID-19 specific IgM antibody and IgG antibody, IgG antibody from negative to positive, or 4 times or more higher for IgG antibody in the recovery period than in the acute phase) were added in the latest seventh edition of the diagnosis and treatment plan, which provided more diagnostic basis for clinical practice (16).

Frankly speaking, without the support of epidemiology, clinical symptoms, laboratory results and positive nucleic acid, typical CT imaging findings of ground-glass changes and distribution areas are difficult to differentiate from other pneumonias in daily work. However, the two cases also have another important commonality, that is, pulmonary lesions developed dynamically in a short period of time (2-3 days). Although the clinical symptoms of patient 2 (the son) were mild, the lung lesions showed further enlargement 3 days later. Therefore, if a patient has typical CT changes with lung lesions rapidly progression in a short time, the clinicians should first suspect COVID-19 in the current situation. And the patient should be isolated immediately, even if he has no clear history of the epidemic or viral nucleic acid results. Although CT can be used for diagnosis, differential diagnosis, dynamic observation of disease changes, prognosis assessment, but its radiation dose problem needs attention. The two patients underwent 5 chest CT examinations respectively during their hospitalization and discharge follow-up, showing the potential harm of radiation. In addition, sometimes CT examination has certain hysteresis, and some patients have clinical and CT nonconformity (17).

In summary, COVID-19 poses a great challenge for global public health $(18,19)$. CT and RT-PCR have their own advantages and disadvantages in fighting against the epidemic. We should make full use of their advantages and adopt other means to make up for their disadvantages. In view of the false negative of RT-PCR, clinicians could try to take samples from multiple sites (especially the lower respiratory tract secretions) to improve its positive rate. CT examination, although not a substitute for nucleic acid as a diagnostic basis, can still play an important role in disease assessment, monitoring, follow-up, but its radiation dose should be taken seriously.

\section{Acknowledgments}

Funding: This study was supported by Guizhou Science and Technology Project \{QKHZC[2020]4Y002\}, Guiyang Science and Technology Project $\{$ ZKXM[2020]4\}, and Guizhou Science and Technology Department Key Lab Project $\{\mathrm{QKF}[2017] 25\}$.

\section{Footnote}

Conflicts of Interest: All authors have completed the ICMJE 
uniform disclosure form (available at http://dx.doi. org/10.21037/apm.2020.03.34). The authors have no conflicts of interest to declare.

Ethical Statement: The authors are accountable for all aspects of the work in ensuring that questions related to the accuracy or integrity of any part of the work are appropriately investigated and resolved. All procedures performed in studies involving human participants were in accordance with the ethical standards of the institutional and/or national research committee(s) and with the Helsinki Declaration (as revised in 2013). Written informed consent was obtained from the patient for publication of this case report and any accompanying images. A copy of the written consent is available for review by the Editor-in- Chief of this journal.

Open Access Statement: This is an Open Access article distributed in accordance with the Creative Commons Attribution-NonCommercial-NoDerivs 4.0 International License (CC BY-NC-ND 4.0), which permits the noncommercial replication and distribution of the article with the strict proviso that no changes or edits are made and the original work is properly cited (including links to both the formal publication through the relevant DOI and the license). See: https://creativecommons.org/licenses/by-nc-nd/4.0/.

\section{References}

1. World Health Organization. Novel Coronavirus (2019$\mathrm{nCoV}$ ) situation reports. Available online: https://www. who.int/emergencies/diseases/novel-coronavirus-2019/ situation-reports

2. Li Q, Guan X, Wu P, et al. Early Transmission Dynamics in Wuhan, China, of Novel Coronavirus-Infected Pneumonia. N Engl J Med 2020. doi: 10.1056/NEJMoa2001316.

3. Zhu N, Zhang D, Wang W, et al. A Novel Coronavirus from Patients with Pneumonia in China, 2019. N Engl J Med 2020. doi: 10.1056/NEJMoa2001017. [Epub ahead of print].

4. Seven days in medicine: 8-14 Jan 2020. BMJ 2020;368:m132.

5. World Health Organization. World experts and funders set priorities for COVID-19 research. Available online: https:// www.who.int/news-room/detail/12-02-2020-world-expertsand-funders-set-priorities-for-covid-19-research

6. WHO. Coronavirus disease (COVID-2019) situation reports. Available online: https://www.who.int/emergencies/ diseases/novel-coronavirus-2019/situation-reports

7. Laboratory testing for coronavirus disease(COVID-19) in suspected human cases. WHO 2020. Available online: https://www.who.int/publications-detail/laboratory- testing-for-2019-novel-coronavirus-in-suspected-humancases-20200117

8. Lin C, Ye R, Xia YL. A meta-analysis to evaluate the effectiveness of real-time PCR for diagnosing novel coronavirus infections. Genet Mol Res 2015;14:15634-41.

9. Kanne JP. Chest CT Findings in 2019 Novel Coronavirus (2019-nCoV) Infections from Wuhan, China: Key Points for the Radiologist. Radiology 2020;295:16-7.

10. Rothe C, Schunk M, Sothmann P, et al. Transmission of 2019-nCoV Infection from an Asymptomatic Contact in Germany. N Engl J Med 2020;382:970-1.

11. Diagnosis and Treatment Plan of Novel Coronavirus Pneumonia (5th edition) (Chinese). 2020. Available online: http://www.nhc.gov.cn/jkj/s3577/202002/a5d6f7b8c48c45 1c87dba14889b30147.shtml

12. Lei J, Li J, Li X, et al. CT Imaging of the 2019 Novel Coronavirus (2019-nCoV) Pneumonia. Radiology, 2020;295:18.

13. Chung M, Bernheim A, Mei X, et al. CT imaging features of 2019 novel coronavirus (2019-nCoV). Radiology 2020;295:202-7.

14. Shi H, Han X, Zheng C. Evolution of CT Manifestations in a Patient Recovered from 2019 Novel Coronavirus (2019-nCoV) Pneumonia in Wuhan, China. Radiology 2020;295:20.

15. Diagnosis and Treatment Plan of Novel Coronavirus Pneumonia (6th editon) (Chinese). 2020. Available online: http://www.nhc.gov.cn/yzygj/s7653p/202002/8334a8326d d94d329df351d7da8aefc2.shtml

16. Diagnosis and Treatment Plan of Novel Coronavirus Pneumonia (7th editon) (Chinese). 2020. Available online: http://www.nhc.gov.cn/yzygj/s7653p/202003/46c9294a7df e4cef80dc7f5912eb1989.shtml

17. Xu YH, Dong JH, An W, et al. Clinical and computed tomographic imaging features of Novel Coronavirus Pneumonia caused by SARS-CoV-2. J Infect 2020. [Epub ahead of print].

18. Chan JFW, Yuan S, Kok KH, et al. A familial cluster of pneumonia associated with the 2019 novel coronavirus indicating person-to-person transmission: a study of a family cluster. Lancet 2020;395:514-23.

19. Mahase E. China coronavirus: what do we know so far? BMJ 2020;368:m308.

Cite this article as: Tang $\mathrm{L}$, Ye Z, Huang Z, Zeng X, Wang T, Xu R, Wang R, Song B. Coronavirus disease 2019 (COVID-19): two case reports from a family cluster. Ann Palliat Med 2021;10(2):2338-2342. doi: 10.21037/apm.2020.03.34 